\title{
ILCEA
}

Revue de l'Institut des langues et cultures

d'Europe, Amérique, Afrique, Asie et Australie

27 | 2016

Approches ergonomiques des pratiques

professionnelles et des formations des traducteurs

\section{La prévention comportementale et contextuelle : intégrer une approche ergonomique dans la formation des traducteurs}

Behavioural and contextual prevention: how to integrate ergonomics into translator training

Verhaltens- und Verhältnisprävention: Ergonomie zum Bestandteil der

Übersetzerausbildung machen

Lucia Peters-Geiben

\section{OpenEdition}

Journals

Édition électronique

URL : http://journals.openedition.org/ilcea/4026

DOI : 10.4000/ilcea.4026

ISSN : 2101-0609

Éditeur

UGA Éditions/Université Grenoble Alpes

Édition imprimée

ISBN : 978-2-84310-336-0

ISSN : $1639-6073$

\section{Référence électronique}

Lucia Peters-Geiben, « La prévention comportementale et contextuelle : intégrer une approche ergonomique dans la formation des traducteurs », ILCEA [En ligne], 27 | 2016, mis en ligne le 08 novembre 2016, consulté le 20 avril 2019. URL : http://journals.openedition.org/ilcea/4026 ; DOI : 10.4000/ilcea.4026

Ce document a été généré automatiquement le 20 avril 2019

(C) ILCEA 


\title{
La prévention comportementale et contextuelle : intégrer une approche ergonomique dans la formation des traducteurs
}

\author{
Behavioural and contextual prevention: how to integrate ergonomics into \\ translator training \\ Verhaltens- und Verhältnisprävention: Ergonomie zum Bestandteil der \\ Übersetzerausbildung machen
}

Lucia Peters-Geiben

\section{Préserver le bien-être tout au long de la vie professionnelle - réflexions générales}

1 En choisissant la profession de traducteur, on ne se soucie guère des conséquences potentiellement néfastes de cette activité sur la santé. Après tout, il ne s'agit pas d'une profession présentant un danger mortel ou causant des accidents du travail

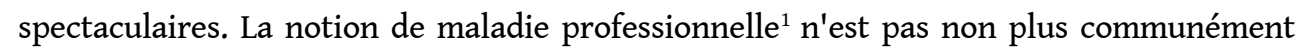
associée à un travail de bureau: une activité centrée sur le traitement de texte est généralement perçue comme un travail léger à cause de la petite amplitude des mouvements et du faible niveau d'activité physique, essentiellement lié à l'effort statique de maintenir une posture (Montreuil, $2008: 13$ ).

2 L'expérience personnelle de l'auteure atteinte de troubles musculo-squelettiques (TMS) ${ }^{2}$ causés par le travail intensif sur écran, des travaux de recherche sur le sujet ainsi que des statistiques d'organismes compétents dressent un tableau bien différent. Ainsi, la Direction générale de l'administration et de la fonction publique (DGAFP) du Ministère de la décentralisation et de la fonction publique constate : «En France, les TMS constituent la première cause de maladie professionnelle reconnue, avec une progression annuelle 
d'environ $18 \%$ depuis 10 ans. Les demandes d'indemnisation pour cause de TMS [...] représentent aujourd'hui $76 \%$ des maladies professionnelles indemnisées » (DGAFP, 2015 : 7). Ce phénomène est également connu sur le plan européen : la cinquième enquête européenne sur les conditions de travail (EWCS) menée en $2010^{3}$ montre que les douleurs du dos (47\%/46\%), du cou, des épaules ou des membres (45\%/41\%) ainsi que les maux de tête et la fatigue oculaire $(46 \% / 33 \%)$ sont les principaux problèmes de santé connus au cours des douze mois précédant l'enquête (Eurofound, 2012).

Selon la Chambre des salariés de Brême en Allemagne, les TMS s'inscrivaient en tête des statistiques sur les causes d'incapacité de travail au début des années 2000 (Arbeitnehmerkammer Bremen, 2003), ce qui est confirmé par le rapport-santé de l'assurance-maladie DAK-Gesundheit 2015 qui, en plus, identifie le secteur de l'administration parmi les trois secteurs subissant le taux le plus élevé d'absentéisme en Allemagne (DAK, 2015).

4 Des travaux de recherche sur la santé des interprètes et traducteurs en Lettonie, présentés en mars 2015 au colloque international Traducteurs à l'œuvre: approches ergonomiques des pratiques professionnelles et des formations de traducteurs à l'université de Grenoble, montrent que les premiers symptômes commencent à se manifester après quelques mois de travail seulement et peuvent évoluer en véritable problème de santé au bout de 5 ans. Tandis que $50 \%$ des répondants disent ne jamais avoir mal aux poignets, $45 \%$ ont répondu « de temps en temps » et $5 \%$ disent qu'ils ressentent souvent ce genre de douleurs (Gizeleza \& Engelsone, 2015).

5 Lorsqu'on prend conscience du nombre de clics de la souris et du nombre de frappes effectués tout au long d'une journée de travail de traducteur, ce résultat n'est pas étonnant. Dans des conditions de laboratoire, Ehrensberger-Dow et Massey (2014b) ont constaté qu'un traducteur professionnel effectue 5,6 clics de la souris et 70,7 frappes par minute, ce qui revient à 33936 frappes et 2688 clics par journée de travail de huit heures. La validité d'une telle extrapolation est bien sûr discutable. Cependant, l'ampleur de ces valeurs est confirmée par un sondage effectué par l'auteure du présent article au sein de la DGT (Direction générale de la traduction) à la Commission européenne en $2015^{4}$ : pour le groupe de traducteurs et traductrices examiné, le nombre moyen de clics par jour de travail s'élève à 2541 (nombre le moins élevé : 55955, le plus élevé : 4543); le nombre moyen de frappes par jour s'élève à 15586 (nombre le moins élevé : 5540, le plus élevé : 38 999).

6 Les TMS ne sont pas les seuls soucis liés au travail intensif sur écran. D'autres troubles, comme la fatigue visuelle, la fatigue des jambes ou des malaises dus à une respiration insuffisante n'apparaissent pas dans les statistiques, mais peuvent autant influencer le bien-être que le mal de dos ou les tendinopathies.

7 Les souffrances des individus ne sont pas négligeables, et cela vaut aussi pour les dommages économiques qui en résultent, notamment une qualité moindre du travail et des absences pour incapacité (Arbeitnehmerkammer Bremen, 2003 ; DAK, 2015 ; DGAFP, 2015). 
Illustration 1. - Une position assise incorrecte est à l'origine de nombreuses douleurs.

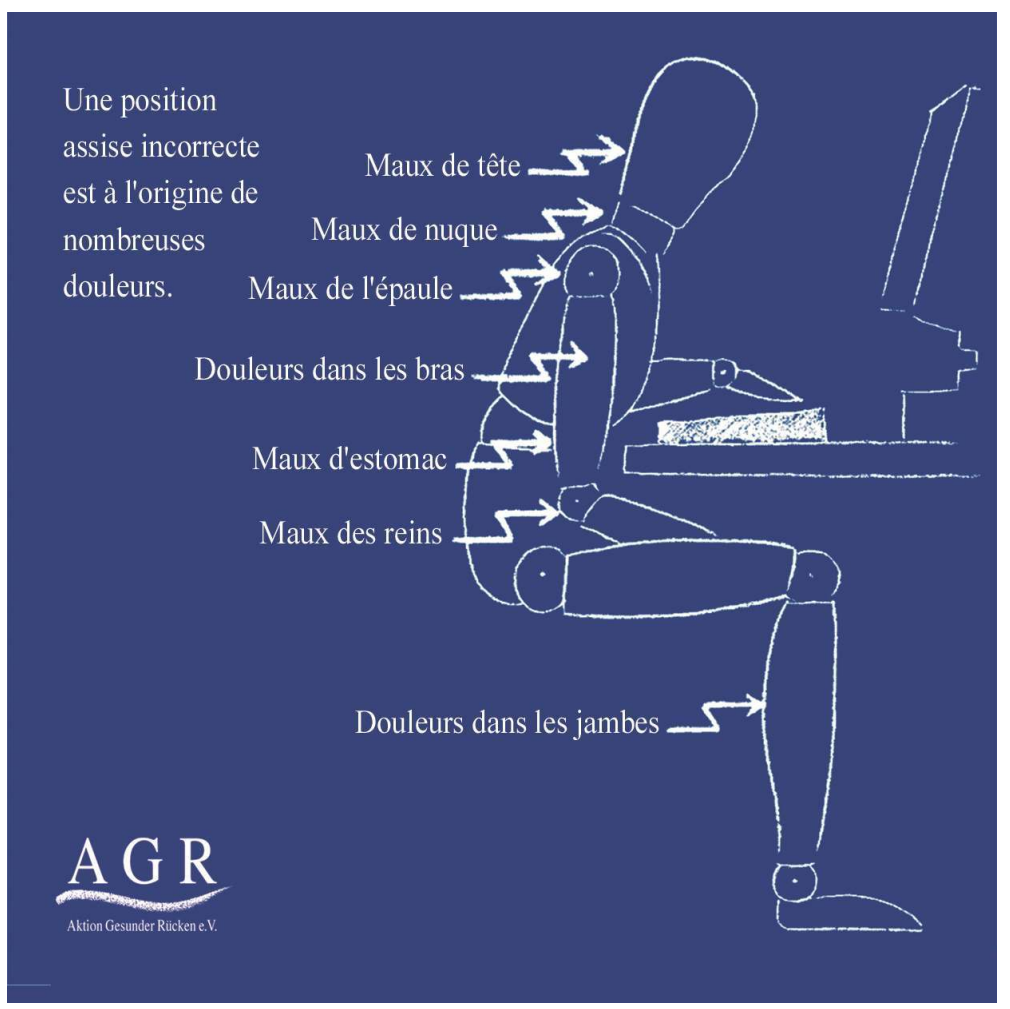

$8 \mathrm{Au}$ vu de ces éléments, intégrer des modules théoriques et pratiques de prévention dans la formation des traducteurs n'est pas simplement une option mais une nécessité. Il est essentiel que les futurs traducteurs intériorisent ces recommandations pendant leurs études universitaires et continuent, consciemment ou non, à les appliquer tout au long de leur vie professionnelle.

Les étudiants ne sont généralement pas sensibilisés à l'ergonomie. Il est urgent de les sortir de leur "innocence » en leur faisant comprendre la nature des risques encourus pour éviter qu'ils les méconnaissent ou qu'ils les minimisent. Le comportement ergonomique est perdu d'avance si on attend le moment de l'entrée dans le monde du travail pour l'appliquer. À ce moment-là, il y a tant d'autres éléments professionnels qui paraissent prioritaires que la préservation du bien-être et de la santé passe au second plan.

10 L'attitude de déni constitue un danger réel, confirmé par les spécialistes : celui qui est en bonne santé se considère trop souvent comme invulnérable (Damany \& Bellis, 2000 : 211).

11 Le présent article aborde essentiellement le côté préventif et montre comment éviter, dès le début, des troubles de santé liés au travail sur écran de visualisation. Les recommandations formulées sont facilement applicables par chacun sans occasionner de coûts particulièrement élevés. Elles se basent d'une part sur l'expérience personnelle de l'auteure, acquise au cours de 30 années de carrière dans le domaine de la traduction, et d'autre part sur la littérature spécialisée publiée entre autres en Allemagne, en France, au Canada et aux États-Unis. La prévention contextuelle est abordée, mais l'accent est mis sur la prévention comportementale puisque celle-ci dépend largement de l'individu et moins de l'employeur ou de la mise à disposition de mobilier sophistiqué. 

pourra pas remplacer un avis médical en cas de problèmes de santé déjà existants. Il s'agit d'explications parfois très simplifiées, puisqu'une étude détaillée dépasserait le cadre prévu et n'est pas le but de cette publication. Damany et Bellis ${ }^{6}$ (2000) expliquent de façon très détaillée les différentes affections RSI ainsi que leurs traitements possibles et énumèrent une série de facteurs prédisposants comme la génétique, l'arthrite, le diabète, mais pour ces auteurs, le facteur prédominant est une attitude « maniaque » vis-à-vis du travail (Damany \& Bellis, 2000 : 50-56). Ils expliquent bien ce qui distingue les affections RSI, qui mûrissent de façon latente et sont parfois mal considérées par les employeurs :

Repetitive strain injury and cumulative disorder [...] can sound like a form of fraud perpetrated by malcontent, malingering employees [...].

RSI is the most complicated and controversial problem of the workplace. RSI is a perplexing mystery because it adds up so slowly that the body has plenty of time to adapt and compensate. [...] When you finally notice symptoms, it is as if a switch were suddenly turned on, despite the fact that the problems probably have been building for as long as two years, perhaps much more.

C'est exactement ce que l'auteure du présent article a vécu en 2007. La progression sournoise de la maladie est expliquée de façon saisissante :

RSI is caused by working in an extremely repetitive fashion, in one position, for years. Your muscles lock up in that position and fatigue, causing your posture to collapse forward. The unnaturally tensed muscles get inflamed and frequently pinch your nerves and blood vessels. [...] The constant repetition, contorted positions, and small range of motion cause tendons to get sticky and inflamed, causing irritation and fluid buildup. [...] To understand why this all happens [...]: muscles, tendons, and bones (collectively the musculoskeletal system) will adapt somewhat to almost any habitual posture and activity. Unfortunately, they will do this with no regard or accommodation whatsoever to the nervous system, which has no such adaptability. Your nerves may be the messengers of the deteriorating state of affairs, or the actual recipients of the damage. (Damany \& Bellis, $2000: 3,5$ et 18)

\section{La prévention contextuelle}

La première prévention consiste à assurer un environnement matériel favorable et adapté à l'individu qui y travaille, entre autres par le choix du bon mobilier de bureau et d'équipement informatique adéquat.

Selon l'association allemande pour la santé du dos, Aktion Gesunder Rücken, «la prévention contextuelle est un module important parmi toutes les démarches préventives et thérapeutiques. Cette prévention signifie qu'il est important que tous les objets et produits utilisés quotidiennement dans l'environnement de vie, de travail et des loisirs soient dotés d'une ergonomie optimale et qu'ils répondent à des exigences médicales pluridisciplinaires. » (AGR, 2014)

\section{L'ergonomie de bureau}

16 La littérature spécialisée en langue française ne fait pas défaut. Le Guide de formation à l'ergonomie de bureau (Montreuil, 2008) s'est avéré être l'ouvrage le plus utile à l'auteure du présent article. Ce guide canadien aborde de façon très structurée et attrayante tous les éléments nécessaires à l'agencement ergonomique d'un poste de travail. Il explique les aspects anatomiques par du texte enrichi d'illustrations et contient même des tableaux synthétiques des différents types de souris ${ }^{7}$ et claviers disponibles sur le marché.

ILCEA, 27 | 2016 


\section{Recommandations de base ${ }^{8}$}

\section{La posture}

17 En posture de base 9 , les bras et les jambes doivent se trouver dans un angle d'au moins $90^{\circ}$ (avant-bras et cuisses en position horizontale), les avant-bras reposent sur la table, les pieds reposent sur le sol (ou sur un repose-pied) et le dossier de la chaise maintient le dos (angle d'assise légèrement supérieur à $90^{\circ}$ ).

\section{Le siège}

18 Il est possible d'utiliser tout siège qui permet la posture décrite ci-dessus. Une chaise est appelée ergonomique quand elle dispose au moins des éléments suivants : des roulettes, une assise réglable en hauteur et un dossier inclinable.

19 Sur le marché, on trouve un choix varié de sièges ergonomiques avec un nombre étonnant de possibilités de réglage. Il vaut mieux rester sur un modèle simple, le plus important étant de comprendre le réglage et que l'ajustement soit facile à faire. Plus il y a de fonctions, plus grand est le risque de mal régler un élément. L'achat d'un siège hyperéquipé peut coûter très cher et bercer l'utilisateur d'illusions, ce qui est l'un des risques majeurs. Ce ne sera pas la chaise qui va préserver son bien-être, ce sera une combinaison de plusieurs éléments.

\section{Le plan de travail}

Toute table qui permet la posture décrite ci-dessus et laisse un espace suffisant pour les jambes peut convenir. Il faut veiller à ce que la table et la chaise soient ajustées correctement. Si le plan de travail est trop haut et non-ajustable, on peut utiliser un repose-pied pour assurer un confort postural et réduire en même temps la compression des jambes. L'idéal est une table dotée d'un ajustement électrique de la hauteur pour permettre de passer de temps en temps de la position assise à la position debout ${ }^{10}$. 
Illustration 2. - Un bureau réglable en hauteur permet plus d'activité physique sur le poste de travail.

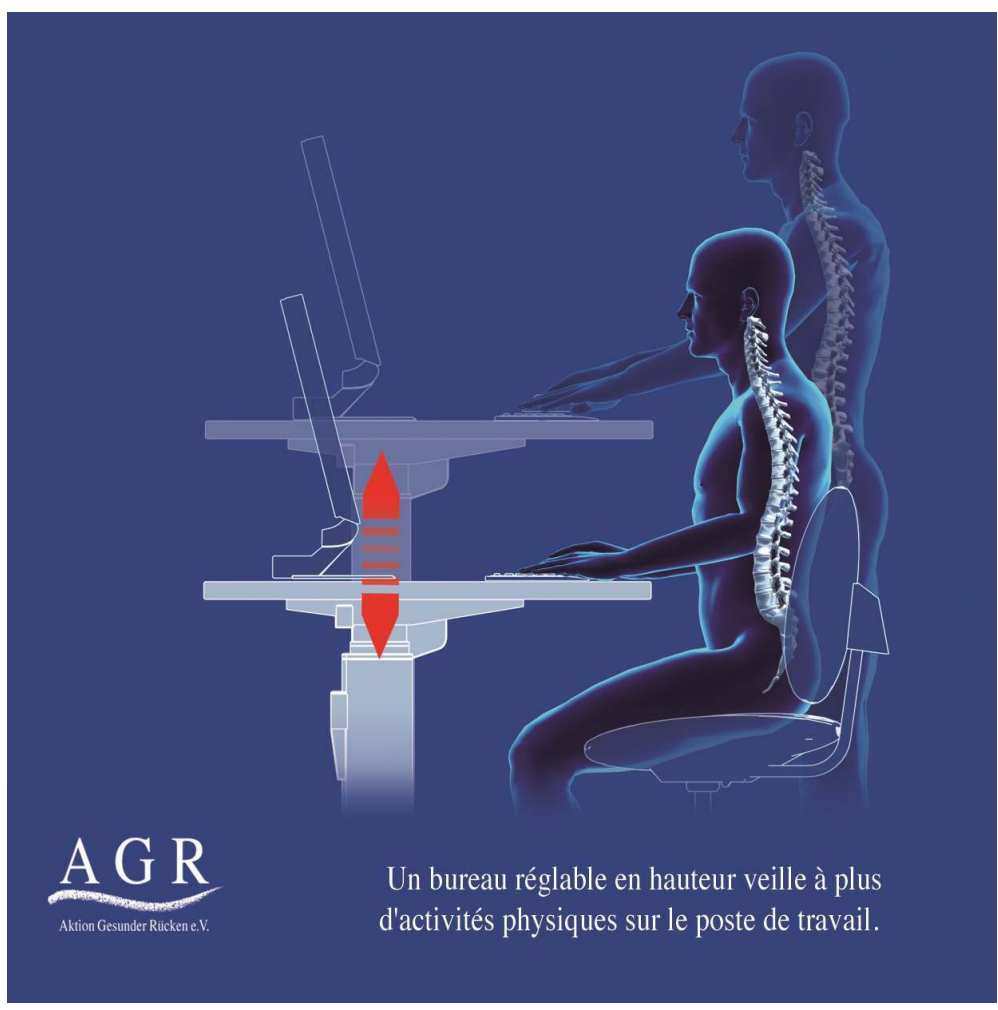

\section{La souris et le clavier}

21 Les souris classiques sont des souris dites horizontales. Pour les utiliser, le poignet et l'avant-bras sortent de leur position naturelle. Les souris dites verticales ont l'avantage que leur utilisation laisse la main et l'avant-bras dans la position neutre, naturelle. Une souris et un clavier traditionnels peuvent tout à fait suffire si on sait comment les utiliser dès le début. Une alternative à la souris est le pavé tactile.

Il y a plusieurs façons de "réconcilier la main et le clavier » (Pineau, 2011: 45), l'une d'elles étant l'utilisation de claviers ergonomiques. Le guide canadien cité plus haut (Montreuil, 2008) présente toutes sortes de claviers disponibles sur le marché. À noter qu'il existe des claviers sans bloc numérique ou dont le bloc numérique est détachable ; ils ont l'avantage que la main manipulant la souris ne doit pas autant s'éloigner du corps, ce qui est moins fatigant pour la musculature du bras et de l'épaule. Le même objectif est encore mieux atteint par les souris du type roller mouse, barmouse ou mouse trapper, placées en position centrale devant le clavier.

\section{Autres éléments}

Ne sont pas abordés ici des éléments comme la lumière et le bruit ambiants, ou la résolution et la luminosité de l'écran. Ceux-ci font néanmoins partie de l'ergonomie physique et sont traités dans les ouvrages de référence (Lancry, 2009 : 24-25).

Toutes les informations nécessaires peuvent être trouvées dans des guides d'ergonomie, disponibles en grand nombre sur Internet et souvent de très bonne qualité. On peut donc 
se demander pourquoi, alors que les informations sur la question sont si facilement accessibles, les troubles résultant du travail intensif sur écran continuent d'augmenter au lieu de diminuer. Doit-on en conclure que la prévention contextuelle, telle que décrite cidessus, n'est pas suffisante pour préserver le bien-être ? Ce sont alors les comportements eux-mêmes qu'il faut interroger, et c'est là l'objectif de la prévention comportementale.

\section{La prévention comportementale}

Selon l'association allemande pour la santé du dos, Aktion Gesunder Rücken, la prévention comportementale comprend les éléments suivants: la description des postures « assis, debout, couché » et des mouvements corporels « se lever, s'asseoir, [...] », des conseils pour un comportement moteur bénéfique pour le dos, la promotion de l'activité physique via les activités du quotidien, par exemple le vélo, le jardinage, ainsi que la promotion de programmes de gymnastique spécifiques (AGR, $2015: 24$ ).

Illustration 3. - Principes du poste de travail de bureau.

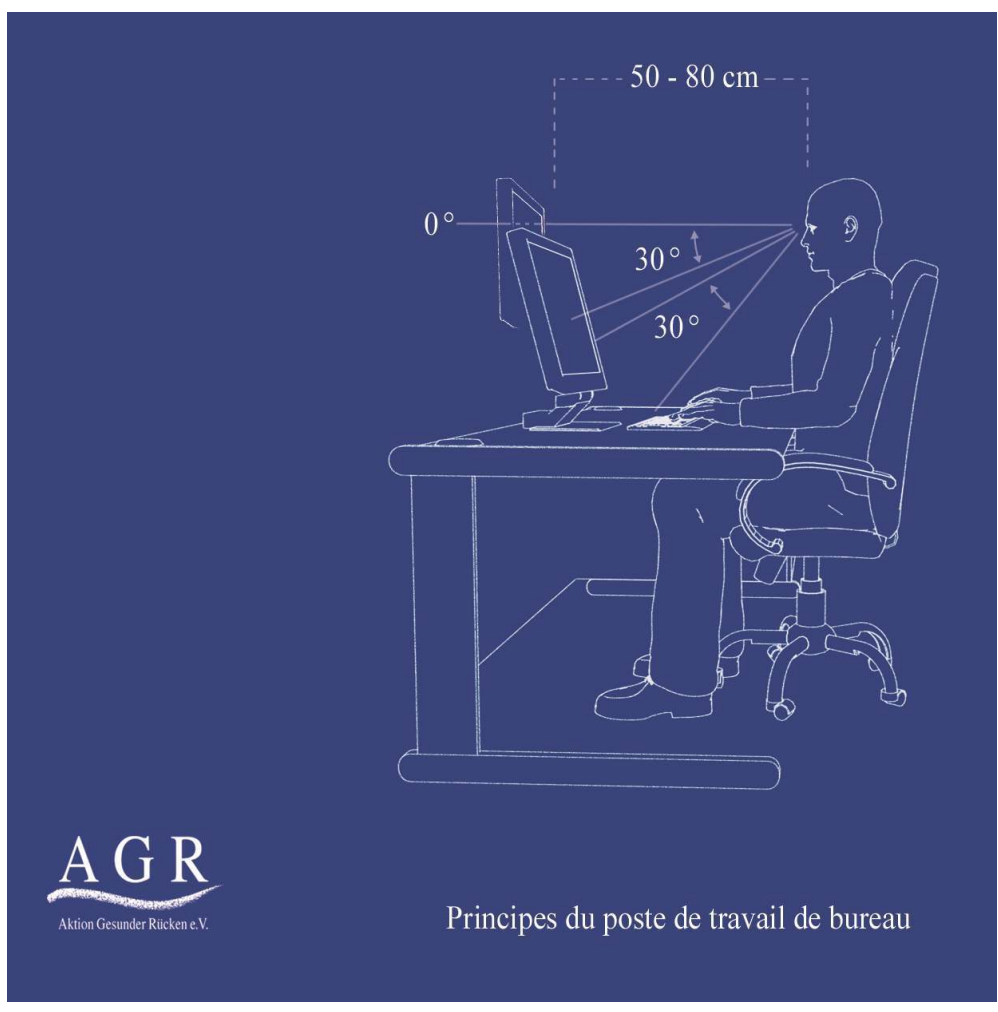

\section{L'utilisation correcte du mobilier et de l'équipement informatique}

Il est évident que le meilleur matériel ne peut atteindre son but à $100 \%$ s'il est mal utilisé. La condition préalable à une utilisation correcte est la connaissance des recommandations de base comme indiquées plus haut. Mais il faut aussi être en mesure de mettre ces recommandations en pratique. 


\section{Le siège} se trouver à la hauteur des yeux ou légèrement en-dessous ${ }^{11}$, sinon on a tendance à incliner la tête vers l'arrière. Même s'il ne s'agit que d'une légère inclinaison, les muscles du cou et des épaules vont le ressentir et se contracter. En plus, en regardant vers le haut, les yeux sont grand ouverts et la fréquence de clignement est réduite, ce qui favorise le syndrome des yeux secs (Montreuil, 2008 : 49 ; Orr, $2007: 58$ ).

Pour chaque chaise, nouvelle ou reprise, l'utilisateur doit pouvoir lire les instructions pour son ajustement. Souvent, les instructions ne sont plus disponibles ou l'utilisateur ne les lit pas et utilise la chaise comme il le juge approprié. L'affichage de ces instructions au plus près du siège devrait être effectué dès l'achat.

Un autre problème est que de nombreuses chaises ergonomiques ne sont pas faciles à ajuster. L'auteure du présent article connaît des chaises quasiment neuves qu'il faut secouer avec force pour pouvoir les régler.

Deux conclusions s'imposent: tout d'abord, à l'achat d'une chaise neuve, il faut, si possible, la tester soi-même en s'asseyant dessus suffisamment longtemps pour pourvoir juger si elle convient ou non aux besoins individuels, et en manipulant toutes les manettes servant à ajuster les différents éléments; ensuite, chaque organisation devrait avoir au moins une personne spécialisée en ergonomie qui pourra donner des avis aux employés et effectuer, si nécessaire, le réglage des chaises. En cas d'acquisition privée, il est recommandé de demander au vendeur si le service après-vente inclut l'entretien ultérieur des mécanismes de réglage et le remplacement de pièces défectueuses.

\section{Le plan de travail}

Il ne faut jamais oublier qu'un plan de travail est une surface froide et dure. En posant le poignet pendant des heures sur une telle surface, la circulation sanguine au niveau des capillaires est gênée. Quand on n'utilise pas ses mains sur le plan de travail pour taper, cliquer, écrire, il faut penser à les reposer sur les jambes qui offrent une surface molle et chaude (Damany \& Bellis, $2000: 69$; Orr, 2007 : 29). À l'instar des employés de bureau des années 1920 qui portaient des protections aux coudes pour éviter une usure rapide de leur chemise, les travailleurs de bureau de notre temps devraient penser à protéger leurs poignets, par exemple avec des chauffe-poignets : on peut trouver des modèles de luxe en angora ou tout simplement utiliser des chaussettes en laine dont on a coupé le pied.

\section{Le PC et l'écran de visualisation}

Ce qui est à éviter à tout prix, c'est de placer l'écran sur le boîtier de l'ordinateur parce que cela limite les possibilités d'ajustement en hauteur. Le bord supérieur de l'écran doit Il faut éviter la position qualifiée de « cou de tortue »: épaules penchées vers l'avant, tête inclinée vers le haut. Le crâne humain ayant un poids moyen de 5,5 kg, l'effort musculaire statique à effectuer dans cette position est beaucoup plus grand.

Si on travaille avec plusieurs écrans, il faut éviter de changer rapidement et de manière répétée d'un écran à l'autre en bougeant la tête brusquement. Cela peut entraver le fonctionnement du système vestibulaire et être à l'origine de vertiges. 


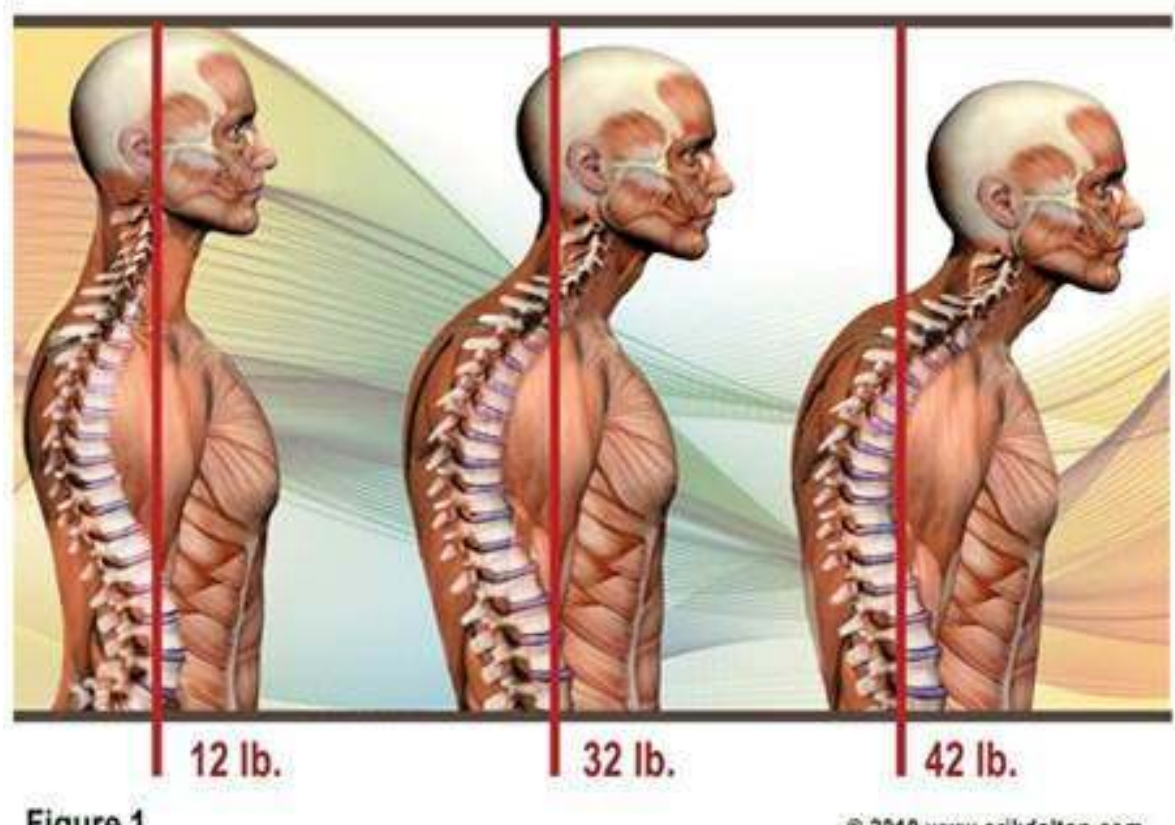

Figure 1

(1) 2010 www.erikdalton.com

\section{La souris}

Les erreurs que l'on peut commettre en utilisant une souris standard sont bien décrites dans l'ouvrage Office Ergonomics (Orr, 2007 : 50-55), qui a permis à l'auteure du présent article d'améliorer son interaction avec la souris standard après avoir été forcée d'utiliser exclusivement une souris ergonomique verticale du type joystick pendant des années. Recommandations de base : choisir une souris adaptée à la taille de la main, poser la paume sur la partie arrondie de la souris de façon à ce que le poignet ne touche pas la surface froide et dure du plan de travail, maintenir main et bras en ligne droite, ne pas éloigner le bras du corps plus que nécessaire, cliquer avec le doigt entier, et non pas seulement avec le bout, et lorsqu'on n'a pas besoin de la souris, retirer la main et la poser sur les jambes, au lieu de continuer à serrer la souris comme si on craignait qu'elle se sauve (Damany \& Bellis, $2000: 205$; Orr, $2007: 27$ ).

Un des meilleurs moyens de prévention des RSI est de remplacer le plus possible la souris, et surtout les doubles-clics, par des alternatives ${ }^{13}$ :

- les raccourcis clavier: ils existent pour beaucoup de logiciels. Il faut prendre le temps d'analyser son propre travail et d'apprendre les raccourcis clavier pour les fonctions utilisées le plus souvent (pour les connaître, pointer avec le curseur sur la fonction voulue et le raccourci s'affiche) ;

- les touches d'accès rapide : appuyer sur la touche ALT et taper le caractère indiqué dans la barre d'outils pour la fonction voulue ;

- une configuration de l'ordinateur de façon à réduire les doubles-clics ${ }^{14}$.

Dans le panneau de configuration (Aide - Centre d'aide et de support), il existe en plus la possibilité d'ajuster la vitesse du double-clic et d'inverser les boutons de la souris ${ }^{15}$.

Pour varier, on peut aussi utiliser des modèles de souris différents et changer plusieurs fois par jour. 


\section{Le clavier} "focalisation externe ", c'est-à-dire que l'on dirige son attention vers ce qui se passe à l'extérieur du corps, et même si on effectue un travail hautement intellectuel, on est coupé de son «moi intérieur » et souvent tellement absorbé que les changements qui ont lieu dans notre corps ne sont pas perçus de façon consciente: mauvaise posture, soif, respiration superficielle, fatigue visuelle, fatigue des jambes, contraction des muscles du cou et des épaules, mains crispées, tout cela échappe au traducteur en pleine concentration sur son texte, jusqu'au moment où sa santé ${ }^{19}$ est déjà considérablement compromise.

Le clavier est loin d'être anodin parmi les causes des TMS dont souffrent les travailleurs de l'écrit. D'après Pineau : «Une formation généralisée à la dactylographie, sur des claviers optimisés par leur distribution de caractères et par leur forme matérielle, ainsi qu'une organisation du travail qui tienne compte de l'aspect répétitif des gestes d'écriture constitueraient déjà une bonne base pour réduire de nombreux problèmes physiques provoqués par l'écriture. » $(2011: 62)$

Savoir utiliser le clavier implique de bien le positionner : une recommandation générale est de toujours poser à plat le clavier pour éviter que les poignets soient en extension, position qui peut entraîner des troubles graves à long terme. Ne pas frapper trop fort non plus, et répartir l'effort entre les dix doigts. Avant l'achat d'un clavier ergonomique, il est fortement conseillé de le tester, l'optimisation des différents types dépendant très fortement des caractéristiques individuelles de l'utilisateur. Contrairement aux souris, les claviers ergonomiques divisent les spécialistes. Une alternative à l'usage du clavier est la reconnaissance vocale ${ }^{16}$, qui offre de nombreux avantages pour les professionnels et peut être associée aux outils de Traduction Assistée par Ordinateur (TAO) sur le poste de travail du traducteur (Ciobanu, 2015).

\section{L'organisation auto-responsable du travail ${ }^{17}$}

Même ceux qui ont fait le bon choix de matériel et qui l'utilisent correctement ne sont pas à l'abri de conséquences néfastes du travail intensif sur écran. La répétitivité des mouvements, la position monotone, le fait d'être absorbé par le travail qui nous fait oublier les besoins de notre corps, tous ces facteurs vont à l'encontre de notre bien-être.

travaille pour un employeur selon les règles de celui-ci, on a une certaine marge de manœuvre pour organiser son travail de façon auto-responsable.

\section{Passer de la focalisation externe à la focalisation interne ${ }^{18}$}

Le corps humain n'est malheureusement pas programmé pour effectuer des pauses actives régulières, comme le font par exemple les ordinateurs qui, après un certain temps d'inactivité, se mettent automatiquement en veille pour protéger l'écran. Au temps des écrans à tube cathodique, c'était une nécessité technique. Pour le corps humain, des pauses régulières et actives sont aussi une nécessité pour rester en bonne santé. 
relâchement » (Montreuil, $2008: 13)$ :

Mais, selon Montreuil :

On aurait tort de qualifier le travail du traducteur de processus inactif car il correspond en fait à une activité professionnelle parfois très éprouvante. Il semble s'agir d'un état quasiment immobile car le travail sur écran implique une position assise statique, avec un travail musculaire dynamique ${ }^{20}$ seulement au niveau des doigts.

Le maintien d'une posture exige que certaines fonctions du corps soient très actives. Trois composantes y sont essentielles : le travail musculaire, la circulation sanguine et la colonne vertébrale. Quelques principes de fonctionnement des membres supérieurs et inférieurs s'appliquent aussi. (2008:13)

Contrairement au travail dynamique des muscles, il s'agit ici «d'une tension sans Lors d'un travail musculaire statique, les muscles sont tendus et la circulation sanguine dans le muscle est réduite : avec le temps, l'entrée du sang est gênée et le sang utilisé s'évacue mal. L'effort est quand même possible grâce à des réserves d'énergie présentes dans le muscle mais, d'une part, cette réserve s'épuise rapidement et, d'autre part, il y a accumulation de déchets de combustion dans le muscle (acide lactique). C'est ce manque éventuel d'énergie et cette accumulation de déchets dans le muscle qui font qu'on ressentira une douleur après un court laps de temps. (Montreuil, $2008: 14$ )

Mais ce n'est pas le seul risque pour la santé. Selon des recherches récentes, le métabolisme se ralentit en position assise prolongée. Les conséquences potentielles à long terme seraient le diabète, les affections cardio-vasculaires, l'obésité et différents cancers, même chez des personnes qui suivent les recommandations de l'Organisation mondiale de la santé (OMS) concernant l'activité physique ${ }^{21}$ (Katzmarzyk, Church, Craig \& Bouchard, 2009 ; van der Ploeg, Chey, Korda, Banks \& Bauman, 2012). Peterson, Sarma et Gordon (2012) confirment ces résultats ainsi que Dunstan et al. (2012) :

of particular importance is the potential for reducing cardiovascular disease risk by briefly breaking up prolonged periods of sitting with activity of at least light intensity. [...] Intervention study findings [...] suggest that the blunted glucose response seen in our study through the inclusion of three brief (2-min) activity breaks per hour during prolonged sitting may ameliorate such consequences [...] (Dunstan et al., 2012 : 980).

Il suffirait donc de se lever et de rester debout quelques minutes et, mieux encore, de marcher et de faire des exercices d'étirement, pour relancer l'activité du métabolisme et décontracter les muscles des jambes en particulier, et de l'ensemble du corps.

Le défi est d'apprendre à passer de la focalisation externe à la focalisation interne régulièrement pour être capable de sentir consciemment les besoins de son corps et de les prendre en compte ${ }^{22}$.

\section{Faire des pauses et maintenir une activité physique}

En règle générale, on a tendance à travailler trop longtemps d'affilée. Celui qui ne dispose pas d'un réveil intérieur, doit trouver des outils d'aide.

51 On peut programmer un logiciel existant, par exemple le calendrier d'un programme de courrier électronique, ou bien utiliser des applications comme Workrave, qui est multilingue et téléchargeable gratuitement sur Internet $^{23}$. Workrave permet la programmation de rappels pour des micro-pauses et pour des pauses de récréation. Pour ces dernières, il propose même des exercices d'étirement. 
52 Selon les résultats de recherche cités plus haut, il convient de bouger toutes les 20 minutes pour maintenir le bon fonctionnement du métabolisme. Ceux qui trouvent cette fréquence trop haute pour interrompre leur travail, peuvent adopter une position assise active $^{24}$. Comme le soulignent Damany et Bellis : «Seated doesn't have to mean sedentary! » $(2000: 177)$.

53 Dans un bureau individuel, on est libre de bouger sur la chaise à son aise, et même lorsqu'on travaille dans un bureau partagé, il y a beaucoup de petits mouvements qu'on peut effectuer dans une réunion sans trop attirer l'attention des autres participants. Mieux encore, on peut choisir une approche militante en expliquant aux autres pourquoi on fait ces mouvements, ce qui contribue à changer les mentalités sur le lieu de travail.

54 Les chercheurs précités ont par ailleurs constaté qu'il n'est pas possible de compenser l'inactivité de toute une journée en faisant seulement du sport pendant les temps de loisirs ; idéalement, on associe les deux. Katzmarzyk, Church, Craig et Bouchard le disent clairement: "Of particular note, the association between sitting time and mortality was independent of leisure time physical activity levels and BMI» (2009).

\section{S'échauffer pour se préparer au travail à l'écran}

En prenant l'habitude de s'échauffer avant de commencer le travail à l'écran, ce n'est pas seulement le corps qui se prépare, c'est aussi l'esprit qui se met en mode de travail. Faire quelques exercices d'étirement, prendre 10 profondes respirations abdominales, serrer et ouvrir les poings 25 fois, masser les avant-bras, se laver les mains avec de l'eau chaude en frottant bien fort, frictionner les mains et avant-bras avec de la crème: chacun peut trouver la préparation qui lui convient le mieux (Damany \& Bellis, 2000 : 192).

\section{Faire de la gymnastique au bureau}

Il existe toute une panoplie d'exercices de gymnastique qui sont praticables au bureau. Un outil exemplaire est le calendrier « En forme sur le lieu de travail » proposé sur le site de l'Association d'assurance accident du Grand-Duché de Luxembourg (AAA, n.d.). Il propose pour chaque jour de travail des séries d'exercices pour toutes les parties du corps.

57 Pour sortir de la position assise, une stratégie assez facile est de saisir toute occasion qui se présente: lorsqu'on parle au téléphone, se mettre en position debout; choisir une imprimante éloignée du poste de travail, etc. 
Illustration 5. - Les changements fréquents entre les positions assises, debout et les déplacements soulagent la colonne vertébrale.

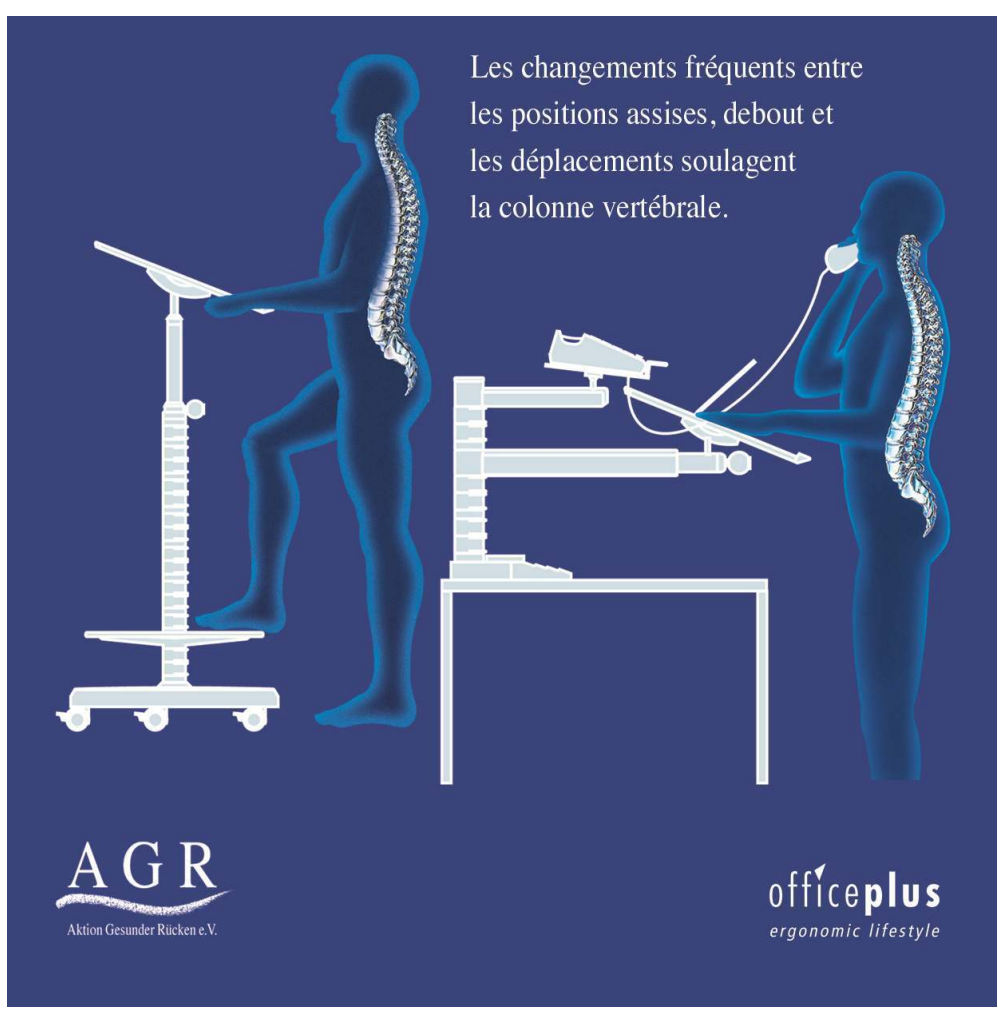

\section{Propositions pour une intégration de l'ergonomie dans la formation des traducteurs}

Pour mettre en œuvre les mesures de prévention décrites ci-dessus dans une organisation ou une institution, il y a deux approches possibles : l'approche descendante et l'approche ascendante. Dans l'un ou l'autre cas, l'action doit être lancée par au moins une personne motivée par la prévention des risques au travail, consciente des enjeux de l'ergonomie pour les futurs traducteurs, et souhaitant intégrer l'ergonomie dans la formation des traducteurs (ci-dessous appelée " porteur de projet »).

\section{L'approche descendante}

59 Si la direction d'une université ou faculté est déjà sensibilisée et reconnaît explicitement l'importance d'un environnement ergonomique pour les étudiants et le corps enseignant ainsi que la nécessité d'enseigner les connaissances et capacités nécessaires pour prévenir les troubles musculo-squelettiques et le syndrome RSI, l'approche descendante a de bonnes chances d'aboutir ${ }^{25}$.

\section{Préliminaires}

60 Le porteur de projet doit tout d'abord vérifier que la direction a la même notion de l'ergonomie que lui et qu'elle a vraiment compris l'enjeu. Malheureusement, les cadres ont souvent tendance à banaliser ce genre de problème ou, dans le pire des cas, à accuser 
les personnels de simulation (Damany \& Bellis, 2000: 7). Certains cadres pensent que l'ergonomie se limite aux chaises et tables de bureau, et ils n'ont peut-être jamais effectué un travail vraiment intensif sur écran comme celui des traducteurs d'aujourd'hui, même s'ils travaillent sur ordinateur eux-mêmes.

61 Un outil très convaincant peut être l'enregistrement du nombre de clics et de frappes par jour avec le logiciel Workrave, déjà mentionné. Le porteur de projet peut l'effectuer d'abord pour lui-même, comparer les chiffres avec ceux trouvés par Ehrensberger-Dow et Massey (2014b) ainsi que par l'auteure du présent article, puis présenter ces chiffres à la direction.

Dans l'enseignement supérieur, l'action dépend fortement des ressources matérielles de l'université : y-a-t-il déjà des chaises ergonomiques, des tables de différentes hauteurs ou même des postes de travail debout? La situation financière permet-elle d'acquérir ce mobilier? Même une direction convaincue posera toute une série de questions avant de se lancer dans ces dépenses.

63 Avant d'entamer des négociations avec la direction, il convient de se faire une image claire de la situation et de développer une stratégie en commençant par des éléments facilement réalisables. Il peut être intéressant d'étudier si l'intégration de l'ergonomie pourrait constituer un avantage concurrentiel dans le classement national ou international des universités.

\section{Acteurs à impliquer}

Le plus efficace est que le projet soit un projet commun d'un groupe de personnes. Sous l'approche descendante, sont concernés :

- la direction,

- le porteur de projet,

- des membres du corps enseignant,

- un ou plusieurs membres de l'administration pour couvrir l'aspect logistique,

- les étudiants.

Il convient de laisser aux différents acteurs une grande liberté d'action pour que ceux-ci s'approprient le projet et laissent libre cours à leur créativité et imagination.

\section{Période d'exécution du projet}

Pour introduire et intégrer l'ergonomie au sens large dans une organisation, il faut compter trois à quatre ans. Il est recommandé au porteur de projet de ne pas commencer trop d'actions au même moment, mais de progresser au fur et à mesure en intégrant les contributions proposées par les autres acteurs. C'est un travail de longue haleine, même avec le soutien de la direction.

\section{Approche ascendante}

67 Si un tel projet ne peut pas être mené de façon officielle avec le soutien de la direction ou si les procédures officielles sont lentes, le porteur de projet peut commencer seul et chercher des «alliés », parmi ses pairs, les membres de l'administration, les étudiants. Il faut trouver quelques personnes pour lesquelles l'ergonomie et le bien-être au travail sont des sujets importants qu'elles veulent voir progresser. C'est toujours avantageux 
d'avoir un groupe composé de gens avec un profil bien différent et des capacités qui peuvent être utiles pour la réalisation des actions.

Il existe un grand nombre d'actions possibles qui sont peu ou pas coûteuses, et que nous détaillons ci-dessous. Une fois toutes ces possibilités explorées, le projet aura probablement déjà gagné une certaine visibilité et il sera plus facile de convaincre la direction d'en faire un projet officiel de l'université et de le doter de moyens financiers.

\section{Actions possibles, descendantes ou ascendantes ${ }^{26}$}

\section{Actions au niveau de la direction :}

- intégrer l'ergonomie du bureau dans les objectifs d'enseignement de l'université ;

- nommer un « correspondant ergonomie » pour l'université ou la composante concernée (pas nécessairement le porteur de projet) ;

- promouvoir le sujet auprès de la présidence de l'université et des ministères de tutelle ;

- planifier un projet officiel avec une demande de moyens financiers ;

- récompenser des initiatives individuelles d'ergonomie, par exemple, par la diffusion de leur action sur l'intranet de l'université et dans d'autres médias.

\section{Actions au niveau de l'administration :}

- faciliter la diffusion de l'information par la mise en place de panneaux d'affichage dédiés aux sujets du bien-être au travail et de l'ergonomie ainsi que par l'intranet ;

- faciliter la diffusion de l'information par l'impression de posters, dépliants et autre matériel utile ;

- mettre à disposition des espaces pour des activités favorisant le bien-être (par exemple, cours de yoga, exercices de gymnastique en groupe ou individuels) ;

- créer un poste de travail modèle avec chaise ergonomique, table ajustable et équipement ergonomique informatique en fournissant des explications détaillées sur ses réglages;

- intégrer une pause active avec des exercices courts d'étirement dans toutes les réunions durant plus de 30 minutes;

- faciliter les réunions debout par l'acquisition de tables hautes;

- développer une collaboration avec des magasins spécialisés de mobilier de bureau et d'équipement informatique ergonomiques afin d'obtenir du mobilier et des équipements modèles à mettre à disposition des personnels administratifs ou enseignants dans un but publicitaire ou bien à tester par les étudiants, soit sur place, soit en prêt.

\section{Actions au niveau des enseignants universitaires :}

- intégrer des recommandations de base sur l'ergonomie dans les cours ;

- intégrer une pause active avec de courts exercices d'étirement dans tous les cours durant plus de 30 minutes;

- promouvoir des mini-exercices à faire en position assise parmi la population universitaire ;

- dédier des cours de traduction à l'ergonomie : aspects technique, législatif, économique et médical ;

- faire développer des posters, dépliants, petits guides par les étudiants ;

- développer un module thématique au sein duquel les étudiants doivent analyser leur travail sur ordinateur avec l'objectif de trouver les raccourcis clavier utiles, de s'approprier des stratégies de recherche efficaces, etc., ou intégrer ceci dans un module déjà existant ; 
- organiser régulièrement de petites conférences pour sensibiliser la population universitaire ;

- organiser un concours de slogans multilingues sur l'ergonomie et/ou le bien-être au travail ;

- chercher des synergies possibles avec d'autres composantes de l'université ou d'autres universités.

\section{Actions au niveau des étudiants :}

- créer des groupes de yoga, de gymnastique, de discussion sur le bien-être ;

- développer des posters, vidéos;

- créer des œuvres d'art dans le cadre d'un concours sur le sujet ;

- faire des propositions pour améliorer l'environnement ergonomique à l'université.

\section{L'ergonomie à la Direction générale de la traduction (DGT) de la Commission européenne} sein de la Direction $\mathrm{R}$-Ressources. L'auteure du présent article a été nommée «Correspondante ergonomie » avec les trois missions suivantes :

- coordonner, avec les services responsables, la mise en œuvre au sein de la DGT d'une politique prenant en compte l'ergonomie et développer les activités et informations qui y sont associées ;

- mener des actions de sensibilisation et d'information du personnel de la DGT à la politique d'ergonomie ;

- créer un réseau de correspondants au sein des départements linguistiques et autres départements de la DGT afin de mieux connaître les besoins spécifiques liés aux divers métiers et de pouvoir y répondre.

L'expérience personnelle de l'auteure, atteinte de troubles RSI graves deux fois pendant sa carrière à la Commission européenne, était à la base des premiers travaux effectués. Toutes les informations utiles, jusqu'alors dispersées sur une multitude de pages intranet, ont été regroupées dans un vade-mecum, qui est régulièrement mis à jour et qui contient de courts résumés sur chaque sujet ainsi que des liens vers les pages intranet concernées.

71 Le développement d'actions sur le plan de la prévention comportementale, et notamment la sensibilisation et l'information (sur les recommandations ergonomiques de base, l'utilisation optimale de l'ordinateur pour éviter les clics-souris, la promotion des exercices d'étirement, un guide de référence rapide pour le travail sur ordinateur...) est un processus d'apprentissage continuel pour l'auteure du présent article: l'analyse bibliographique ainsi que la collaboration étroite avec, entre autres, le médecin du travail de la Commission européenne lui a permis de corriger des erreurs d'appréciation et de développer des actions fondées. Plusieurs de ces actions ont été intégrées dans un nouveau programme pluriannuel transversal de santé et de bien-être de la Commission appelé fit@work.

La sensibilisation et l'information se font via différents canaux : l'intranet offre un vaste éventail d'informations souvent très détaillées; des posters sont placés dans les couloirs pour promouvoir l'activité physique et sensibiliser sur le sujet de l'ergonomie; des formations ${ }^{27}$ sont données régulièrement ainsi que des conférences à l'heure du déjeuner ; la bibliothèque de la DGT est équipée de toute une série d'ouvrages pertinents 
ainsi que d'un kiosque qui regroupe toutes les informations en un endroit unique et où on peut tester de l'équipement ergonomique. Une boîte fonctionnelle de courrier électronique (DGT-ERGONOMICS) est diffusée via tous ces canaux et reçoit régulièrement des courriels avec des questions et suggestions, même de la part de collègues d'autres services de la Commission européenne.

En ce qui concerne la prévention contextuelle, la DGT dispose du mobilier ergonomique standard mis à disposition par la Commission européenne. Pour son réglage et agencement, les collègues peuvent faire appel à la correspondante ergonomie, à des collègues du domaine de la logistique ou au service médical. Sur demande justifiée et approuvée par le service médical, le service logistique peut fournir des tables électriquement réglables en hauteur. Quelque $15 \%$ du personnel, traducteurs et autres catégories de personnel, utilisent des souris ergonomiques verticales.

$\mathrm{Vu}$ que chaque individu est unique, non pas seulement en ce qui concerne la taille, l'état de santé et les facteurs éventuels de prédisposition, mais aussi dans sa manière de ressentir les choses, de penser et d'agir, l'ergonomie n'est pas un sujet facile et ne propose pas de remède miracle. Bien au contraire, le changement de mentalité, l'acceptation de situations ressenties comme négatives, la modification des attitudes, tout cela s'avère difficile. Chacun a besoin d'un encouragement continuel et d'un soutien moral, pratique et pragmatique, de son environnement de travail (et de l'environnement privé également).

Nous pensons qu'il est important d'enseigner les bases de l'ergonomie de bureau et un comportement respectant les besoins du corps le plus tôt possible, dès l'école maternelle et primaire, et surtout plus tard pendant les études universitaires ou autres, qui conduisent à une activité professionnelle « informatisée » et sédentaire.

En conclusion, le bien-être au bureau repose sur des notions identifiables et applicables : auto-responsabilité, activité physique, mouvement, attention juste, micro-pauses fréquentes. Quant à l'intégration de l'ergonomie au sens large du terme dans une organisation, elle requiert beaucoup de motivation, de persévérance, de créativité, et une démarche axée sur la communication et la sensibilisation.

\section{BIBLIOGRAPHIE}

AAA - Association d'assurance accident (n.d.), En forme sur le lieu de travail, en ligne sur le site du ministère de la Sécurité sociale et du ministère du Travail, de l'Emploi et de l'Économie sociale et solidaire, Luxembourg : <www.aaa.lu/publications/calendrier-en-forme-sur-le-lieu-de-travail>. AKTION GESUNDER RÜCKEN - AGR (2014), Médecins et thérapeutes certifiés, en ligne sur <www.agrev.de/fr/medecin-et-therapeutes/medecins-et-therapeutes-certifies>.

AKTION GESUNDER RÜCKEN - AGR (2015), cours à distance : De la prévention comportementale vers la prévention contextuelle, Selsingen, Allemagne.

ARBEITNEHMERKAMMER BREMEN (2003), Bildschirmarbeit und Gesundheitsrisiken - Argumente für ergonomisch gestaltete Bildschirmarbeit, Info-Brief $\mathrm{n}^{\circ} 09$, en ligne sur < 
www.arbeitnehmerkammer.de/cms/upload/Downloads/

Info_Mitbestimmung_und_Technologieberatung/Bildschirmarbeit_und_Gesundheitsrisiken.pdf $>$.

BriÈre Julien, Fouquet Natacha, HA Catherine, Imbernon Ellen, Plaine Julie [...] Valenty Madeleine (2015), Des indicateurs en santé travail. Les troubles musculo-squelettiques du membre supérieur en France, Saint-Maurice : Institut de veille sanitaire.

Ciobanu Dragoş (2015, mars), « There be Dragons - myths and realities about Automatic Speech Recognition systems for professional translators ", communication présentée au colloque Traducteurs à l'œuvre: approches ergonomiques des pratiques professionnelles et des formations de traducteurs, Grenoble, France.

DAK-GESUNDHEIT (2015), DAK-Gesundheitsreport 2015, en ligne sur <www.dak.de/dakonline/live/ dak/download/Vollstaendiger_bundesweiter_Gesundheitsreport_2015-1585948.pdf>.

DAMANY Suparna \& BELLIS Jack (2000), It's Not Carpal Tunnel Syndrome!: RSI Theory and Therapy for Computer Professionals, États-Unis : Simax, résumé en ligne sur <s91524683.onlinehome.us/rsi/>.

DiRECTION GÉNÉRALE DE L'ADMINISTRATION ET DE LA FONCTION PUBLIQUE - DGAFP (2015), Guide pratique Démarche de prévention des troubles musculo-squelettiques (TMS), ministère de la Décentralisation et de la fonction publique, en ligne sur <www.fonction-publique.gouv.fr/files/files/publications/ coll_outils_de_la_GRH/guide_pratique_TMS.pdf>.

Dunstan David W., Kingwell Bronwyn A., LaRSEn Robyn, Healy Genevieve N., CeRIN Ester [...] O WEN Neville (2012), « Breaking Up Prolonged Sitting Reduces Postprandial Glucose and Insulin Responses », Diabetes Care, 35(5), 976-983, <dx.doi.org/10.2337/dc11-1931>.

EHRENSBERGER-Dow Maureen \& MASSEY Gary (2014), « Translators and machines: working together ", Man vs. Machine?, 1, 199-207, Proceedings of XXth World Congress of the International Federation of Translators.

EUROFound (2012), Fifth European Working Conditions Survey, Luxembourg : Publications Office of the European Union, <dx.doi.org/10.2806/34660>.

EUROSTAT (2000), Statistiques européennes des maladies professionnelles (EODS) - Phase 1 Méthodologie (Doc. OS/E3/HSW/2000/1081), Luxembourg : Commission européenne.

GiZELEZA Helena \& ENGELSONE Larisa (2015, mars), « Interpreters' and translators' occupational health and safety in Latvia », communication présentée au colloque Traducteurs à l'œeuvre: approches ergonomiques des pratiques professionnelles et des formations de traducteurs, Grenoble, France.

Katzmarzyk Peter T., Church Timothy S., CRaig Cora L. \& Bouchard Claude (2009), « Sitting Time and Mortality from All Causes, Cardiovascular Disease, and Cancer », Medicine \& Science in Sports \& Exercise, ACSM Journal, 41(5), 998-1005, <dx.doi.org/10.1249/MSS.0b013e3181930355>.

LANCRY Alain (2009), L'ergonomie, Paris, France : Presses universitaires de France.

MonTREUIL Sylvie (2008), Ergonomie : Travail de bureau avec écran de visualisation - Guide de formation, Département des relations industrielles de l'Université Laval, Canada, en ligne sur <cgsst.com/ stock/fra/guide-dergonomie.pdf>.

OMS - Organisation mondiale de la santé (1946), « Préambule à la Constitution de l'Organisation mondiale de la santé », Conférence internationale sur la Santé, New York, États-Unis.

OMS - Organisation mondiale de la santé (2016), Aide-mémoire $N^{\circ} 385$, en ligne sur <www.who.int/ mediacentre/factsheets/fs385/fr/>. 
ORR Susan (2007), Office Ergonomics: Preventing Repetitive Motion Injuries \& Carpal Tunnel Syndrome, Lafayette, Canada : Letsdoyoga.com.

Peterson Mark D., SARMa Aruna V. \& Gordon Paul M. (2012), « Sitting Time and All-Cause

Mortality Risk », Archives of Internal Medicine, 172(16), 1270-1272, <dx.doi.org/10.1001/

archinternmed.2012.2527>.

PINEAu Martine (2011), « La main et le clavier : histoire d'un malentendu », ILCEA (14), <

ilcea.revues.org/1067>.

Ploeg Van Der Hidde P., Chey Tien, Korda Rosemary J., Banks Emily \& Bauman Adrian (2012), « Sitting Time and All-Cause Mortality Risk in 222497 Australian Adults », Archives of Internal Medicine, 172(6), 494-500, <dx.doi.org/10.1001/archinternmed.2011.2174>.

\section{NOTES}

1. Sous les codes M700 (ténosynovite de la main et du poignet), M770 (épicondylite médiale - coude) et M771 (épicondylite latérale - coude), la méthodologie des Statistiques européennes sur les maladies professionnelles (EODS) cite quelques affections souvent diagnostiquées en relation avec le travail intensif sur écran. Les troubles du type RSI (Repetitive Strain Injuries) dus aux mouvements répétitifs devraient aussi faire partie de ce groupe. À noter : Les traducteurs n'étant pas pris en compte spécifiquement dans la liste des professions, ils pourraient se retrouver inclus dans les groupes 40 «Employés de type administratif » ou 41 "Employés de bureau " (Eurostat, 2000).

2. Brière, Fouquet, Ha, Imbernon, Plaine, Rivière et al. (2015) en donnent la définition suivante : "Les troubles musculo-squelettiques (TMS) recouvrent un large ensemble d'affections périarticulaires qui touchent les tissus mous (muscles, tendons, nerfs, vaisseaux, cartilages) et se traduisent principalement par des douleurs et une gêne fonctionnelle souvent quotidiennes. » Ces troubles sont également appelés « pathologies d'hyper-sollicitation ».

3. Enquête menée dans 34 pays européens auprès de 44000 travailleurs; valeurs entre parenthèses données pour femmes/hommes.

4. Enregistrement des données à l'aide du logiciel Workrave, téléchargeable gratuitement sur Internet ; prise en compte d'une journée entière de travail $(8 \mathrm{~h})$ de 10 traducteurs volontaires en conditions de travail réelles.

5. Ce traducteur est un passionné des raccourcis clavier et a établi une liste d'environ 250 raccourcis utiles.

6. Suparna Damany a un Master of Science Physical Therapy et est certifiée pour la physiothérapie de la main et l'évaluation ergonomique; Jack Bellis est éditeur multimédia, utilisateur IT de longue date et, lors de la rédaction du livre, en phase de convalescence.

7. Au type de "souris horizontale» il faut par exemple ajouter la souris verticale de type «joystick» sur laquelle le clic gauche est effectué avec le pouce, une vraie alternative pour ceux dont les tendons de l'index sont devenus trop sensibles.

8. Ces recommandations valent pour des personnes non encore atteintes de troubles musculosquelettiques/RSI. En cas de problèmes, il faut consulter un médecin ou thérapeute spécialisé.

9. On verra plus bas que la variation de la posture et l'assise active sont des éléments importants de prévention.

10. En règle générale, une journée de travail devrait compter au maximum $50 \%$ du temps en position assise, $25 \%$ en position debout et $25 \%$ de marche (AGR, $2015: 255$ ). L'utilisation d'un appuie-fesses peut être utile. On en trouve dans les magasins spécialisés, mais aussi dans les grandes surfaces en tant qu'accessoires pour les tables de repassage. Si on utilise un ordinateur 
portable, on peut facilement se procurer d'un poste de travail debout, par exemple en remodelant une étagère existante, en achetant une table haute ou même en mettant un carton stable sur la table pour y poser le portable.

11. En cas d'utilisation de lunettes avec verres progressifs, il convient de positionner l'écran plus bas pour que le cou soit droit ou légèrement incliné vers le bas.

12. Schéma disponible en ligne sur le site d'Erik Dalton : <erikdalton.com/> (20 juillet 2016).

13. Les explications se réfèrent à Windows 7 et Office 2010 .

14. Ouvrir l'explorateur Windows (par exemple avec le raccourci clavier touche Windows $+\mathrm{E}$ ) : onglet Outils - Options des dossiers ou onglet Affichage - Options ; sous l'onglet Général, cochez : Ouvrir les éléments par simple clic (sélection par pointage).

15. Attention! Si on change simplement la souris de main quand on éprouve des douleurs au poignet, on risque d'avoir, à moyen terme, les mêmes problèmes de l'autre côté. Il faut essayer de connaître la cause des douleurs, se faire soigner et changer le comportement, l'équipement ou même les deux.

16. À l'achat d'un tel logiciel, il faut prévoir une période d'entraînement. Sous Windows 7 , il y a un élément de reconnaissance vocale pour contrôler l'ordinateur avec la voix, qui figure dans le panneau de configuration.

17. Le contenu de cette partie est basé sur des informations et recommandations que l'auteure a reçues de la part de médecins, physiothérapeutes, etc., consultés pour tendinopathies devenues chroniques.

18. Ce paragraphe est basé sur un entretien avec le psychologue diplômé et enseignant de Yoga K. Geiben, pratiquant sous le nom Gangaputra en Allemagne dans la région de Francfort.

19. L'Organisation mondiale de la santé définit la santé comme suit: «La santé est un état de complet bien-être physique, mental et social, et ne consiste pas seulement en une absence de maladie ou d'infirmité. » (OMS, 1946)

20. «L'effort dynamique (de mouvement) [...] est caractérisé par une alternance de tension et de relâchement des muscles. » (Montreuil, $2008: 14$ )

21. Recommandations de l'OMS : «Les adultes de 18 à 64 ans devraient pratiquer au moins 150 minutes hebdomadaires d'une activité physique d'intensité modérée, ou au moins 75 minutes hebdomadaires d'une activité physique intense, ou une combinaison équivalente d'activité physique d'intensité modérée à forte ; pour en retirer des bienfaits supplémentaires en matière de santé, les adultes devraient porter à 300 minutes par semaine la pratique d'une activité physique d'intensité modérée ou l'équivalent; des activités de renforcement musculaire mettant en jeu les principaux groupes de muscles devraient être pratiquées deux jours par semaine ou plus. » (OMS, 2015)

22. À l'aide du jeu de mot anglais « mindful or mind full », on peut facilement expliquer de quoi il s'agit: avoir l'attention juste, la pleine conscience, lors de toute activité. Pour en faire partie intégrante de sa vie, il n'est pas nécessaire d'étudier à fond les courants philosophiques qui prônent cette prise de conscience de l'instant présent. On peut commencer en l'appliquant aux petites activités journalières, autrement passées inaperçues dans la conscience.

23. Après le téléchargement, il vaut mieux changer les intervalles préprogrammés des rappels, sinon on reçoit un rappel toutes les trois minutes, ce qui peut être assez dérangeant. Le logiciel a été conçu pour prévenir les troubles RSI et prend en compte le temps passé à l'activité de clavier et de souris pour calculer les intervalles : <www.workrave.org/media/leaflet/pdf/leaflet-fr.pdf>.

24. Le site <www.agr-ev.de> donne des informations sur les sièges de bureau actifs. Le ballonsiège, conçu pour y faire des exercices légers, destinés à la stimulation des réflexes neurophysiologiques, est une alternative bon marché qui peut remplacer la chaise pendant des périodes courtes (AGR, 2015).

25. Pour Damany et Bellis, l'approche descendante est la seule qui a vraiment des chances d'aboutir. (2000: 214) C'est vrai pour un programme d'entreprise, mais il ne faut en aucun cas 
décourager les initiatives individuelles ou locales dès le début; celles-ci peuvent se révéler particulièrement efficaces et peuvent contribuer à un changement de mentalité envers l'ergonomie au bénéfice du bien-être des employés.

26. Les propositions d'actions sont reprises ensemble dans ce paragraphe et il s'agit bien entendu d'une liste non-exhaustive.

27. Le module de formation principale d'une heure et demie avec des informations et des exercices est régulièrement complet. Le taux de satisfaction de $95 \%$ calculé sur base d'un questionnaire rempli par les participants après la formation est très encourageant, mais ne dit rien, bien entendu, sur la mise en œuvre effective des conseils.

\section{RÉSUMÉS}

Le rôle toujours plus important de l'ordinateur dans le quotidien des traducteurs les oblige régulièrement à rester assis de manière prolongée à leur poste de travail en posture statique, ce qui peut être à l'origine de troubles divers. Cet article explique quelques principes ergonomiques de base qu'il est important de connaître et de mettre en pratique.

Les recommandations concernent la prévention contextuelle, entre autres le bon choix du mobilier de bureau et de l'équipement informatique, ainsi que la prévention comportementale, qui se traduit par l'utilisation correcte de cet équipement et par la prise de précautions pour préserver le bien-être tout au long de la journée de travail et au-delà. Une troisième partie porte sur l'élaboration d'une approche pour l'intégration de l'ergonomie dans la formation des traducteurs, du point de vue théorique et pratique, et dans une perspective de durée.

The versatility of the PC regularly keeps translators sitting at their desks in a static posture for long periods, which may give rise to various complaints. This article explains the basic principles of office ergonomics every translator should know and apply.

Recommendations deal with contextual prevention, including the choice of adequate office furniture and IT peripherals, as well as with behavioural prevention. The latter comprises the correct use of the equipment and the application of precautionary measures to preserve one's well-being throughout the working day and beyond. The third section of the article attempts an approach for the integration of ergonomics into the training of translators, both in theory and practice and with long-lasting effect.

Mit dem Computer als Hauptarbeitsmittel verbringen Übersetzer den Großteil ihrer Arbeitszeit in statischer Sitzhaltung am Schreibtisch. Der vorliegende Artikel beschäftigt sich mit den ergonomischen Grundsätzen für Bildschirmarbeitsplätze, die alle Übersetzer kennen und anwenden sollten.

Die Empfehlungen beziehen sich zum einen auf die Verhältnisprävention, u. a. die Wahl geeigneter Büromöbel und Peripheriegeräte, zum anderen auf die Verhaltensprävention. Hier geht es um die korrekte Verwendung dieser Ausstattung sowie um Vorkehrungen zum Erhalt des Wohlbefindens über den gesamten Arbeitstag hinweg und darüber hinaus. Der dritte Teil des Artikels enthält Ansätze dafür, wie das Thema Ergonomie in Theorie und Praxis sowie mit dem Ziel der Langzeitwirkung in die Übersetzerausbildung integriert werden kann. 
INDEX

Keywords : office ergonomics; behavioural prevention; contextual prevention; vertical mouse; posture; active sitting; physical activity; well-being; internal focus; external focus; selfresponsibility; standing workstation; variation

Schlüsselwörter : Ergonomie im Büro; Verhaltensprävention; Verhältnisprävention; vertikale Maus; Haltung; aktives Sitzen; Bewegung; Wohlbefinden; interner Fokus; externer Fokus; Selbstverantwortung; Steharbeitsplatz; Abwechslung

Mots-clés : ergonomie du bureau, prévention comportementale, prévention contextuelle, souris verticale, posture, position assise active, activité physique, bien-être, focalisation externe, focalisation interne, auto-responsabilité, poste de travail debout, variation

\section{AUTEUR}

\section{LUCIA PETERS-GEIBEN}

Correspondante ergonomie

Direction générale de la traduction, Commission européenne 